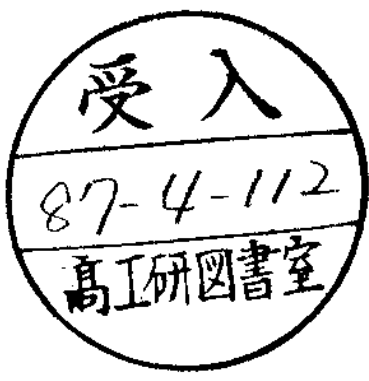

\title{
OPERATOR ORDERING AND SUPERSYMMETRY
}

V. de Alfaro

Dipartimento di Fisica Teorica, Universita di Torino INFN, Sezione di Torino

and

I.C.T.P., Trieste

S. Fubini

CERN - Geneva

G. Furlan

Dipartimento di Fisica Teorica, Universita di Torino INFN, Sezione di Torino

and

I.C.T.P., Trieste

and

M. Roncade $\left.11 i^{*}\right)$

CERN - Geneva

\section{A B S T R A C T}

We investigate supersymmetric quantum mechanics in curved space. In particular we discuss, in the framework of the canonical formulation, the role of supersymmetry (SUSY) and of invariance under general co-ordinate transformations (G.C.T.) in solving the operator ordering ambiguity. It is shown that G.C.T. invariance is instrumental to achieve such a result. We also examine the connection with the Weyl ordering of operators and the relevance of our results for the path integral approach.

*) On leave from Dipartimento di Fisica Nucleare e Teorica, Universita di Pavia. 


\section{INTRODUCTION}

The quantization of a particle in a curved space is a problem that has been with us for many years [1]. In this introductory section we shall first offer a brief survey of the subject in its development and then we shall illustrate the main points and new results of our investigation.

The classical Lagrangian for a system of $N$ real coordinates $x^{\mu}(t)$ on a manifold 16 with metric $g_{\mu \nu}(x)$ reads, in absence of potential

$$
L=\frac{1}{2} g_{\mu \nu}(x) \dot{x}^{\mu} \dot{x}^{\nu}, \mu, \nu=1 \ldots N,
$$

and leads to the Hamiltonian

$$
H=\frac{1}{2} g^{\mu \nu}(x) p_{\mu} p_{\nu}
$$

At the quantum level there is a crucial problem: the non vanishing commutator of $\times \mu$ and $p_{\nu}$ introduces an ordering ambiguity. We recall that a similar problem arises, in a milder form, for the quantization of a particle in an external electromagnetic field. (It can be interesting to remark that this latter problem can be imbedded in the above general case by using the Kaluza-Klein higher dimensional procedure for the description of the electromagnetic field).

At the classical level the Lagrangian (1.1) exhibits invariance under general coordinate transformations (G.C.T.)

$$
x^{\prime \mu}=F^{\mu}(x)
$$

The fundamental requirement of form invariance of $H$ under G.C.T. has been recognized to be connected with the choice of the ordering by $B$ Dewitt in a classical paper [2]. He showed that the invariant Hamiltonian is given by

$$
H_{0}=\frac{1}{2} b_{\mu}-g^{\mu \nu}(x) b_{\nu}^{+}
$$

with

$$
p_{\mu}^{+}=p_{\mu}+\frac{i}{4} \partial_{\mu} \ln g=g^{1 / 4} p_{\mu} g^{-1 / 4}
$$


$-2-$

$$
p_{\mu}^{-}=p_{\mu}-\frac{i}{4} \partial_{\mu} \ln g=g^{-1 / 4} p_{\mu} g^{1 / 4} \text {. }
$$

He also proved that the most general invariant Hamiltonian has the form

$$
H=H_{0}+C R
$$

for any constant $c, R$ being the curvature.

Anyway the G.C.T. invariant Hamiltonian is uniquely defined for a flat space $(R=0)$. As well known, the ordering problem is present in this case also.

If one starts from a cartesian basis

$$
g_{\mu \nu}^{(0)}=\delta_{\mu \nu}
$$

and performs a G.C.T., a metric is introduced on the (constant curvature) manifold

$$
g^{\mu \nu}(x)=\frac{\partial F^{\mu}}{\partial x^{\alpha}} \frac{\partial F^{\nu}}{\partial x^{\alpha}}
$$

In the path integral approach to quantization one may wonder where lies the trouble corresponding to the ordering ambiguity of the canonical approach since there are no operators and commutators. The point is that the action integral in the functional context is, in general, not Riemannian, but depends in an essential way on the detailed form in which the discrete sum is performed. This goes under the name of Ito integral. Let us consider, for example, the expression

$$
g_{\mu \nu}(\xi)\left(x_{1}-x_{2}\right)^{\mu}\left(x_{1}-x_{2}\right)^{\nu}
$$

which appears in the expansion of the action in terms of small coordinate and time differences. The three choices

$$
\begin{aligned}
& \xi=x_{1}, \\
& \xi=x_{2}, \\
& \xi=\frac{x_{1}+x_{2}}{2},
\end{aligned}
$$


turn out to be not equivalent and lead to different results that are the exact counterpart of the ordering ambiguity for the canonical quantization. The connection is established by a result of Berezin [3] stating that the path integral obtained by the middle point choice defines the same quantum theory as the operator Hamiltonian where the products of $x$ and $p$ are symetrized; this is the so called weyl ordering (W.0.) prescription [4].

It is interesting to note that the expression (1.4) is not W.o. For instance in the case of flat space the difference $\mathrm{H}_{0}-\left(\mathrm{H}_{0}\right)_{\text {wo }}$ is given by the compact expression [5]

$$
H_{0}-\left(H_{0}\right)_{w \Delta}=\Delta V=\frac{1}{8} \Gamma_{\mu \nu}^{\lambda} \Gamma_{\lambda s}^{\mu} g^{\nu \rho}
$$

where $\Gamma_{\mu v}^{\lambda}$ is the Christoffel symbol induced by the G.C.T. as in (1.7).

The problem outlined above is of particular physical interest. Indeed the quantum description of a relativistic spinless particle in a gravitational field exhibits similar features [6]. In turn this can be of relevance to the string case where, as emphasized by Maharana and Veneziano [7], the Fradkin-Polyakov treatment leads to analogous ordering problems [8].

A new element in these considerations is represented by the introduction of supersymmetry which is expected to shed new light on the ordering problem. In fact it is known that for supersymetric theories (in flat space) the ordering of the boson operators determines the corresponding form of the fermionic operators [9].

The matter has been examined by several authors [10]: in those works however the interplay between supersymmetry and general coordinate invariance has not been discussed in detail.

In this paper we offer a systematic analysis of the ordering problem based on the combined use of supersymmetry (SUSY) and invariance under general coordinate transformations (G.C.T.). Since the matter is rather subtle we prefer to approach the problem in the canonical operator formalism. This does not exclude that a functional treatment can be rather interesting.

The main ingredient of our work is representes by the introduction, suggested by supersymmetry, of two sets of momenta $p_{\mu}^{(1)}$ and $p_{\mu}^{(2)}$ which can be considered as the natural generalization of the harmonic oscillator annihilation and creation operators, " $a$ " and $a^{+}$. They will be instrumental 
in proving the general coordinate invariance of the whole approach and in expressing the fundamental dynamical variables in a weyl ordered form.

In Section (2) we discuss the supersymetric formulation of the model, while chapter ( 3 ), still at the classical level, introduces the canonical formalism, the fundamental supersymmetry charges and the Hamiltonian. Chapter (4) contains a discussion of quantization: we shall see that supersymetry (contrary to a common belief) still allows an ambiguity in the ordering of the operators. This is eliminated if one adds, as we do in chapter (5), the requirement of general coordinate invariance, which thus plays the key role in selecting the unique form of the quantum Hamiltonian.

The main features related to the weyl ordering of operators are dealt with in chapter (6): it is shown that, unlike the non supersymmetric case, the unique, general coordinate invariant Hamiltonian is Weyl ordered when the appropriate variables are used.

In the concluding chapter (7) we point out the relevance of our results to the path integral approach.

Some auxiliary calculations and examples are presented in the Appendices A), B), C).

2. THE SUPERFIELD FORKULATION .

We denote by $Q_{1}$ and $Q_{2}$ the two SUSY generators which satisfy the algebra

$$
\begin{aligned}
& \left\{Q_{1}, Q_{2}\right\}=2 H, \\
& \left\{Q_{1}, Q_{1}\right\}=\left\{Q_{2}, Q_{2}\right\}=0 .
\end{aligned}
$$

Consider a set of real superfields

$$
\phi^{\mu}(t)=x^{\mu}(t)+i \bar{\psi}^{\mu}(t) \theta+i \psi^{\mu}(t) \bar{\theta}+F^{\mu}(t) \theta \bar{\theta},
$$

to be viewed as coordinates of a $N$-dimensional manifold $W G(1 \leq \mu \leq N)$, endowed with a metric tensor $g_{\mu \nu}(P), P \subset W 6 . \times \mu$ and $F \mu$ are real bosonic variables while $\psi, \Psi$ are complex, anticommating fermions. 
$-5-$

By introducing the explicit representations of the SUSY generators in superspace

$$
\begin{aligned}
& \hat{Q}_{1}=-\frac{\partial}{\partial \bar{\theta}}-i \theta \frac{\partial}{\partial t}, \\
& \hat{Q}_{2}=\frac{\partial}{\partial \theta}+i \bar{\theta} \frac{\partial}{\partial t},
\end{aligned}
$$

the transformation law of $\phi^{\mu}$ can be written as

$$
\delta \phi^{\mu}=-i\left(\xi_{1} \hat{Q}_{1}+\xi_{2} \hat{Q}_{2}\right) \phi^{\mu}
$$

where $\xi_{1}, \xi_{2}$ are infinitesimal, anticomuting parameters. Going to the component fields, one obtains the transformation rules

$$
\begin{aligned}
& \delta_{1} x^{\mu}=\xi_{1} 4^{\mu}, \quad \delta_{1} F^{\mu}=i \xi_{1} \dot{\psi}^{\mu}, \\
& \delta_{1} \psi^{\mu}=0, \quad \delta_{1} \bar{\psi}^{\mu}=\xi_{1}\left(i \dot{x}^{\mu}-F^{\mu}\right) . \\
& \delta_{2} \times^{\mu}=\bar{\psi}^{\mu} \xi_{2}, \quad \delta_{2} F^{\mu}=i \xi_{2} \dot{\bar{\psi}}^{\mu}, \\
& \delta_{2} \psi^{\mu}=\xi_{2}\left(-i \dot{x}^{\mu}-F^{\mu}\right), \quad \delta_{2} \bar{\psi}^{\mu}=0
\end{aligned}
$$

Consider next the superfield

$$
\Lambda=\frac{1}{2} g_{\mu \nu}(\phi) \hat{D}_{1} \phi^{\mu} \widehat{D}_{2} \phi^{\nu}-W(\phi),
$$

where $D_{1}$ and $D_{2}$ denote the covariant derivatives in superspace

$$
\begin{aligned}
& \hat{D}_{1}=-\frac{\partial}{\partial \theta}+i \theta \frac{\partial}{\partial t} 1 \\
& \hat{D}_{2}=\frac{\partial}{\partial \theta}-i \bar{\theta} \frac{\partial}{\partial t} .
\end{aligned}
$$

$W(\phi)$ is a scalar function on $W G$ (its lowest component is the superpotential $W(x)$ of the model). 
$-6-$

It is meaningful to reexpress $\Lambda$ as

$$
\Lambda=A+i S_{1} \theta+i S_{2} \bar{\theta}+L \theta \bar{\theta}
$$

with the new components given by:

$$
\begin{aligned}
& A=\frac{1}{2} g_{\mu \nu}(x) \psi^{\mu} \Psi^{\nu}-W(x), \\
& S_{1}=\frac{1}{2} g_{\mu \nu}(x)\left(F^{\mu}+i \dot{x}^{\mu}\right) \Psi^{\nu}-\partial_{\lambda} w(x) \bar{\psi}^{\lambda}+ \\
& +\frac{1}{2} \partial_{\lambda} g_{\mu \nu}(x) \bar{\psi}^{\lambda} \psi \mu \Psi^{\nu} \\
& S_{2}=\frac{1}{2} g_{\mu \nu}(x)\left(F^{\mu}-i \dot{x}^{\mu}\right) \psi^{\nu}-\partial_{\lambda} \omega(x) \psi^{\lambda}+ \\
& +\frac{1}{2} \partial_{\lambda} g_{\mu \nu}(x) \psi \lambda \psi \mu \Psi^{\nu} . \\
& L=\frac{1}{2} g_{\mu \nu}(x) \dot{x}^{\mu} \dot{x} \nu^{\nu}+\frac{1}{2} g_{\mu \nu}(x)\left(\bar{\psi} \mu \dot{\psi}^{\nu}-\dot{\bar{\psi}} \mu \psi \nu\right) \\
& -\frac{1}{2} \partial_{\lambda} g_{\mu \nu}(x)\left(\bar{\psi}^{\lambda} \psi^{\mu}-\bar{\psi}^{\mu} \psi^{\lambda}\right) \dot{x}^{\nu}-V(x, \psi, \bar{\psi}, F) \text {. }
\end{aligned}
$$

Finally the quantity $V$, which embodies all the dependence of the Lagrangian on the auxiliary fields $F$, has the form

$$
\begin{aligned}
& V(x, \psi, \bar{\psi}, F)=\Gamma_{\lambda, \mu \nu} F^{\lambda} \psi^{\mu} \Psi^{\nu}-\frac{1}{2} g_{\mu \nu}(x) F^{\mu} F^{\nu}+ \\
& +\partial_{\lambda} w(x) F^{\lambda}-\partial_{\lambda} \partial_{\sigma} W(x) \psi^{\lambda} \bar{\psi}^{\sigma}+ \\
& +\frac{1}{2} \partial_{\lambda} \partial_{\sigma} g_{\mu \nu}(x) \psi^{\lambda} \bar{\psi}^{\sigma} \psi_{\mu} \bar{\psi}^{\nu}
\end{aligned}
$$

and $\Gamma_{\mu \nu}^{\lambda}$ is the christoffel symbol.

since $\Lambda$ is a real superfield $\left(\Lambda^{+}=\Lambda\right)$ the rules of transformation for the components can be easily derived as in the case of $\phi \mu$. 
- $7-$

From

$$
\delta \wedge=-i\left(\xi_{1} \hat{Q}_{1}+\xi_{2} \hat{Q}_{2}\right) \wedge
$$

it follows that

$$
\begin{aligned}
& \delta_{1} A=\xi_{1} S_{2}, \quad \delta_{1} S_{1}=\xi_{1}(i \dot{A}-L), \\
& \delta_{1} S_{2}=0, \quad \delta_{1} L=i \xi_{1} \dot{S}_{2} . \\
& \delta_{2} A=-\xi_{2} S_{1}, \quad \delta_{2} S_{1}=0, \\
& \delta_{2} S_{2}=-\xi_{2}(i \dot{A}+L), \quad \delta_{2} L=i \xi_{2} \dot{S}_{1} .
\end{aligned}
$$

The susY invariant action is finally

$$
I_{\text {suse }}=\int d t \int d \theta d \bar{\theta} \wedge \text {. }
$$

Clearly $L$ is the Lagrangian dictated by the superfield formulation of a susY invariant theory. Yet, we will work in the component formalism. As it will be seen, the analysis will take a particularly simple form by making use of Lagrangians which differ from L by a total time derivative.

A glance at the transformation properties displayed in eds. $(2.11 \mathrm{a}, \mathrm{b})$ shows indeed the possibility of having two equivalent Lagrangians whose transformation properties are particularly simple.

By defining

$$
\begin{aligned}
& L_{1} \equiv L-i \dot{A}=\frac{1}{2} g_{\mu \nu}(x) \dot{x} \dot{x}^{\nu}+i g_{\mu \nu}(x) \bar{\psi}^{\mu} \dot{\psi}^{\nu}+ \\
& +i \Gamma_{\lambda, \mu \nu} \dot{x}^{\nu} \bar{\psi}^{\lambda} \psi^{\mu}+i \partial_{\lambda} \omega(x) \dot{x}^{\lambda}-V(x, \psi, \bar{\psi}, F)
\end{aligned}
$$

and

$$
\begin{aligned}
& L_{2} \equiv L+i \dot{A}=\frac{1}{2} g_{\mu \nu}(x) \dot{x} \mu \dot{x}^{\nu}+i g_{\mu v}(x) \psi^{\mu} \dot{\psi}^{\nu}- \\
& -i \Gamma_{\lambda, \mu \nu} \dot{x}^{\nu} \bar{\psi}^{\mu} \psi^{\lambda}-i \partial \lambda \omega(x) \dot{x}^{\lambda}-r(x, \psi, \bar{\psi}, F),
\end{aligned}
$$


it turns out that

$$
\begin{aligned}
& \delta_{1} L_{1}=0, \quad \delta_{2} L_{1}=2 i \xi_{2} \dot{S}_{1} \\
& \delta_{1} L_{2}=2 i \xi_{1} \dot{S}_{2}, \quad \delta_{2} L_{2}=0 .
\end{aligned}
$$

The relation between the two Lagrangians is

$$
L_{2}-L_{1}=2 i \dot{A}
$$

We notice from their definitions that $L_{1}$ and $L_{2}$ are not real but rather $\mathrm{L}_{1}^{+}=\mathrm{L}_{2}$.

In spite of this fact (of course not affecting the equations of motion) their role will result particularly useful as it will emerge from the developments of the next section discussing the canonical formulation of the model.

In view of this it is useful to proceed to the elimination of the auxiliary variables $F \mu$, as can be done by using the equations of motion. One has

$$
F^{\mu}=g^{\mu \nu} \partial_{\nu} W+T_{s \sigma}^{\mu} \psi^{s} \bar{\psi}^{\sigma} .
$$

Accordingly the potential $r(x, 4, \bar{\psi}, F)$ takes on the form

$$
\begin{aligned}
\hat{V}(x, q, \bar{\psi})=\frac{1}{2} g^{\mu \nu} \partial_{\mu} w \partial_{\nu} w & +D_{\mu} D_{v} w \bar{\psi} \psi \psi^{\nu}+ \\
& +\frac{1}{2} R_{\mu v \rho \sigma}+\mu \bar{\psi}^{\nu} \psi s \bar{\psi}^{\sigma}
\end{aligned}
$$

$$
D_{\mu} D_{\nu} w \equiv D_{\mu} \partial_{\nu} w=\partial_{\mu} \partial_{\nu} w-\Gamma_{\mu v}^{\sigma} \partial_{\sigma} w(x)
$$

and $R_{\mu \nu \lambda \rho}$ is the Riemann curvature tensor constructed with $g_{\mu \nu}$ (for all the definitions we follow the book by Weinberg [11]).

The on-shell transformation rules read

$$
\begin{aligned}
& \delta_{1} x^{\mu}=\xi_{1} \psi^{\mu}, \quad \delta_{1} \psi^{\mu}=0, \\
& \delta_{1} \bar{\psi}^{\mu}=\xi_{1}\left(i \dot{x}^{\mu}-\partial^{\mu}\left(x+\Gamma_{\rho \sigma}^{\mu} \bar{\psi}^{s} \psi^{\sigma}\right) .\right.
\end{aligned}
$$


$-9-$

$$
\begin{aligned}
& \delta_{2} x^{\mu}=-\xi_{2} \Psi^{\mu}, \quad \delta_{2} \bar{\psi}^{\mu}=0, \\
& \delta_{2} \psi^{\mu}=-\xi_{2}\left(i \dot{x}^{\mu}+\partial^{\mu} w-\Gamma_{\rho \sigma}^{\mu} \Psi^{\rho} \psi^{\sigma}\right) .
\end{aligned}
$$

We conclude the section by recalling the simplified formulation which is based on the use of the vierbein fields $e_{\mu}^{a}(x)$. This will result particularly advantageous in the ensuing discussions because one can then employ fermionic variables which are invariant under reparametrizations. "vierbeine" can be introduced via the definition ${ }^{(*)}$

$$
g_{\mu \nu}(x)=\sum_{1}^{N} a e_{\mu}^{a}(x) e_{\nu}^{\alpha}(x),
$$

$\Sigma_{a} e^{\mu} e_{\mu b}=\delta_{a b}$

Correspondingly the desired fermionic quantities are

$$
\psi^{a} \equiv e_{\mu}^{a} \psi^{\mu}, \quad \Psi^{a} \equiv e_{\mu}^{a} \Psi^{\mu}
$$

and in these variables $L$ reads

$$
\begin{aligned}
L & =\frac{1}{2} g_{\mu \nu}(x) \dot{x}^{\mu} \dot{x}^{\nu}+\frac{i}{2}\left(\psi^{a} \dot{\psi}^{a}-\dot{\psi}^{a} \bar{\psi}^{a}\right)- \\
& -i \omega_{\mu, a b} \dot{x}^{\mu} \bar{\psi}^{a} \psi^{b}-\hat{v}\left(x^{\mu}, \psi^{a}, \bar{\psi}^{a}\right),
\end{aligned}
$$

where the spin connection $\omega_{\mu, a b}$ is defined as

$$
\omega_{\mu, a b}=-e_{a \lambda} \partial_{\mu} e_{b}^{\lambda}-e_{a \nu} \Gamma_{\mu \lambda}^{\nu} e^{\lambda} b .
$$

Having in mind the same motivation which led us to introduce $L_{1}$ and $L_{2}$ starting from L expressed in terms of $\psi^{\mu}, \bar{\Psi}^{\mu}$, one can likewise consider two

(*) For simplicity the flat index "a" is referred to a compact case. 
$-10-$

new Lagrangian $L_{I}$ and $L_{I I}$ out of $L$ expressed in terms of $4^{a}, \overline{4}^{a}$. These are

$$
\begin{aligned}
L_{I} \equiv & L-i \dot{B}=\frac{1}{2} g_{\mu \nu}(x) \dot{x}^{\mu} \dot{x}^{\nu}+i \bar{\psi}^{a} \dot{\psi}^{a}- \\
& -i \omega_{\mu, a b} \dot{x}^{\mu} \bar{\psi}^{a} \psi^{b}-\hat{V}\left(x^{\mu}, \psi^{a}, \bar{\psi}^{a}\right), \\
L_{\underline{I I}} \equiv & L+i \dot{B}=\frac{1}{2} g_{\mu \nu}(x) \dot{x}^{\mu} \dot{x}^{\nu}+i \psi^{a} \dot{\psi}^{a}- \\
& -i \omega_{\mu, a b} \dot{x}^{\mu} \bar{\psi}^{a} \psi^{b}-\hat{v}\left(x^{\mu}, \psi^{a}, \bar{\psi}^{a}\right),
\end{aligned}
$$

where

$$
B=\frac{1}{2} 4^{a} \Psi^{a}
$$


$-11-$

3. THE CANONICAL APPROACH.

The analysis carried out in the previous Section suggested that supersymmetry is most simply realized in terms of the Lagrangian $\mathrm{L}_{1}$ and $\mathrm{L}_{2}$. Therefore, on our way to quantization, we shall find rewarding to consder two independent canonical formulations stemming from $\mathrm{L}_{1}$ and $\mathrm{L}_{2}$ beside the one based on the Lagrangian $L$. This will allow us to derive for the fundamental suse charges $Q_{1}$ and $Q_{2}$ two meaningful expressions, which have the remarkable advantage of retaining their simplicity in the quantized case.

Let us therefore start by considering the canonical formalism arising from $\mathrm{L}_{1}$. The bosonic and fermionic momenta are:

$$
p_{\mu}^{(1)} \equiv \frac{\partial L_{1}}{\partial \dot{x}^{\mu}}=g_{\mu \nu}(x) \dot{x}^{\nu}+i \partial_{\mu} W^{\prime}+i T_{\lambda, \mu \nu} \bar{\psi}^{\lambda} \psi^{\nu}
$$

and

$$
i \bar{\psi}_{\mu} \equiv \frac{\partial L_{1}}{\partial \dot{\psi}^{\mu}}=i g_{\mu \nu}(x) \bar{\psi}^{\nu}
$$

respectively $(\% / \partial \dot{\psi} \mu$ acts as a "right" derivative).

Thus in the present case the set of bosonic and fermionic coordinates and momenta is $\Omega_{\mu}\left(x^{\mu}, p_{\mu}^{(1)} ; \psi^{\mu}, i \bar{\psi}^{\mu}\right)$. Their transformation rules under supersymmetry are particularly simple in the case $\xi_{2}=0$ ie

$$
\begin{array}{ll}
\delta_{1} \times^{\mu}=\xi_{1} 4^{\mu}, & \delta_{1} p_{\mu}{ }^{(1)}=0, \\
\delta_{1} 4^{\mu}=0, & \delta_{1} \bar{\psi}_{\mu}=i \xi_{1} p_{\mu}{ }^{(1)} .
\end{array}
$$

Let us turn to the canonical counterpart based on $\mathrm{L}_{2}$. The bosonic and fermionic momenta are

$b_{\mu}^{(2)}=\frac{\partial L_{2}}{\partial \dot{x}^{\mu}}=g_{\mu \nu}(x) \dot{x}^{\nu}-i \partial_{\mu} W+i T_{\lambda, \mu \nu} \psi^{\lambda} \Psi^{\nu}$,

$-i \psi_{\mu} \equiv \frac{\partial L_{2}}{\partial \dot{\psi}^{\mu}}=-i g_{\mu \nu}(x) \psi^{\nu}$.

$\partial / \partial \dot{\psi}^{\mu}$ is a left-derivative).

The set of canonical variables is thus $\Omega_{2}\left(x_{\mu}, P_{\mu}(2) ; F^{\mu}, i \psi_{\mu}\right)$ with transformation properties which are remarkably simple in the case $\xi_{1}=0$ : 
$-12-$

$\delta_{2} \times^{\mu}=-\xi_{2} \bar{\psi}^{\mu}, \quad \delta_{2} p_{\mu}^{(2)}=0$

$\delta_{2} \bar{\psi}^{\mu}=0 \quad, \delta_{2} \psi_{\mu}=-i \xi_{2} p_{\mu}^{(2)}$.

The variables $p_{\mu}^{(1)}$ and $p_{\mu}^{(2)}$ are not real, rather $p_{\mu}^{(1)}=p_{\mu}^{(2)+}$, but they will nevertheless play a fundamental role in the subsequent discussion.

More precisely, the usefulness of the canonical treatments based on the two sets of variables $\Omega_{1}$ and $\Omega_{2}$ starts to become apparent when expressing the susY charges $Q_{1}$ and $Q_{2}$ in Hamiltonian form. Consider $Q_{1}$ first. The best choice for the Lagrangian to be used in the Nether theorem is $L_{1}$, owing to its invariance under the transformations generated by $Q_{1}, \delta_{1} L_{1}=0$

Taking then into account the form of $L_{1}$ and of the supersymetry transformations ( 3.3$)$, the Noether theorem yields

$$
Q_{1}=p_{\mu}^{(1)} \psi^{\mu} \text {. }
$$

One proceeds in the same way in determining the form of $Q_{2}$ : by applying the Noether theorem to $\mathrm{L}_{2}$, invariant under the transformations generated by $Q_{2}\left(\delta_{2} L_{2}=0\right)$, we easily get

$$
Q_{2}=p_{\mu}^{(2)} \overline{4}^{\mu} \text {. }
$$

In view of quantization, it is important to notice that the variables occurring in $Q_{1}$ have vanishing Poisson brackets and the same occurs in the case of $Q_{2}$. This is one of the main virtues of our canonical procedure.

We proceed next to derive the canonical variables relevant to the description in terms of $\mathrm{L}_{I}$ and $\mathrm{L}_{I I}$. The bosonic momentum is the same in both cases and reads

$$
p_{\mu} \equiv \frac{\partial L_{I}}{\partial \dot{x}^{\mu}}=\frac{\partial L_{I}}{\partial \dot{x}^{\mu}}=g_{\mu \nu}(x) \dot{x}^{\nu}-i \omega_{\mu, a b} \bar{\psi}^{a} \psi^{b} \text {. }
$$

Notice that $p_{\mu}$ is real $\left(p_{\mu}^{+}=p_{\mu}\right)$ to be contrasted with $p_{\mu}^{(1)}$ and $p_{\mu}^{(2)}$ which are complex objects. Thus $p_{\mu}$ appears as the natural canonical momentum to be quantized. The fermionic momenta corresponding to $\mathrm{L}_{I}$ and $\mathrm{L}_{\text {II }}$ are 
$-13-$

$\frac{\partial L_{I}}{\partial \dot{\psi}^{a}}=i \bar{\psi}_{a}$

$\frac{\partial L_{I}}{\partial \dot{\bar{\psi}}^{a}}=-i \psi_{a}$.

We thus introduce two new sets of bosonic and fermionic coordinates and momenta $\Omega_{I}\left(x^{\mu}, p_{\mu} ; \psi^{a}, i \bar{\psi}_{a}\right)$ and $\Omega_{\pi}\left(x^{\mu}, p_{\mu} ; \bar{\psi}^{a},-i \psi_{a}\right)$. emerging from the canonical formulation based on $L_{I}$ and $L_{I I}$ respectively.

In the following there will be no need to distinguish any further between $\Omega_{I}$ and $\Omega_{I}$ and we shall refer to a single set $\Omega\left(x^{\mu}, b_{\mu} ; \psi^{a}, \bar{\psi}^{a}\right)$.

Let us now provide the explicit form of the Hamiltonian. We have

$$
H=H_{0}+\hat{v}
$$

where

$$
H_{0}=\frac{1}{2} g_{\mu \nu}(x) \dot{x}^{\mu} \dot{x}^{\nu}
$$

and

$$
\begin{aligned}
& \hat{V}(x, \psi, \bar{\psi})=\frac{1}{2} g^{\mu \nu}(x) \partial_{\mu} w \partial_{\gamma} w+D_{\mu} D_{v} w \bar{\psi}^{\mu} \psi^{\nu}+ \\
& +\frac{1}{2} R_{\mu v \lambda_{3}} \bar{\psi}^{\mu} \psi^{v} \bar{\psi}^{\lambda} \psi^{s} .
\end{aligned}
$$

The Hamiltonian can be finally expressed in terms of the three sets of variables $\left(\Omega_{1}, \Omega_{2}, \Omega\right)$. It is easy, by using the appropriate definitions, to write $\hat{V}_{\text {in }}$ terms of the $\left(\psi^{\mu}, \Phi_{\mu}\right),\left(\bar{\psi}^{\mu}, \psi_{\mu}\right),\left(\psi^{\alpha}, \bar{\Psi}^{a}\right)$ pairs. For what concerns $\mathrm{H}_{0}$ we shall use the form

$$
H_{0}=\frac{1}{2} g^{\mu \nu}(x) \pi_{\mu} \pi_{\nu}
$$

where

$$
\begin{aligned}
\pi_{\mu}=g_{\mu \nu}(x) \dot{x}^{\nu} & =p_{\mu}^{(1)}-i \Gamma_{\lambda, \mu \nu} \bar{\psi}^{\lambda} \psi^{\nu}-i \partial_{\mu} w \\
& =p_{\mu}^{(2)}-i T_{\lambda, \mu \nu} \psi^{\lambda} \bar{\psi}^{\nu}+i \partial_{\mu} w \\
& =p_{\mu}+i \omega_{\mu, a b} \bar{\psi}^{a} \psi^{b}
\end{aligned}
$$


$-14-$

according to the set of variables we want to select.

As $f$ ar as the supersymmetry charges $Q_{1}$ and $Q_{2}$ are concerned we shall use in all our discussions the remarkably simple expressions given in eq. (3.8) and (3.9); we therefore skip the Nether theorem derivation based on $\mathrm{L}_{I}$ or $\mathrm{L}_{\mathrm{II}}$.

Let us summarize our findings. Three sets of canonical variables have been selected $\Omega_{1}\left(x^{\mu}, p_{\mu}^{(1)} ; \psi^{\mu}, i \bar{\psi}_{\mu}\right), \Omega_{2}\left(x^{\mu}, p_{\mu}^{(2)} ; \bar{\psi}^{\mu},-i \psi_{\mu}\right), \Omega_{1}\left(x^{\mu}, p_{\mu} ; \psi^{a}, \overline{4}^{a}\right)$, each one providing a particularly simple and convenient expression for one of the generators $Q_{1}, Q_{2}$ and $H$ respectively. The use of one or of another set will be dictated by convenience, according to the particular aspect to be stressed. One immediately ascertains that the three sets have the same Poisson brackets and they are therefore canonically equivalent, connected by a complex canonical transformation. We report here the simple relations among the bosonic momenta $p_{\mu}{ }^{(1)}, p_{\mu}{ }^{(2)}, p_{\mu}$ on which we shall focus some of the arguments for the quantum case. From the explicit expressions of $L_{,} L_{1}$ and $\mathrm{L}_{2}$ one finds

$$
\begin{aligned}
& p_{\mu}^{(2)}-p_{\mu}^{(1)}=i \partial_{\mu} g_{\lambda s} \psi^{\lambda} \bar{\psi}^{3}-2 i \partial_{\mu} w, \\
& p_{\mu}^{(1)}-p_{\mu}=i e_{b}^{\nu} \partial_{\mu} e_{\nu a} \bar{\psi}^{a} \psi^{b}+i \partial_{\mu} w, \\
& p_{\mu}^{(2)}-p_{\mu}=-i e_{a}^{\nu} \partial_{\mu} e_{\nu b} \bar{\psi}^{a} \psi^{b}-i \partial_{\mu} w .
\end{aligned}
$$


4. Quantization .

In order to carry on the quantization procedure we shall replace the variables of the set $\Omega\left(x^{\mu}, p_{\mu} ; \psi^{a}, \bar{\psi}^{a}\right)$ by linear operators - denoted by the same symbols - acting in the Hilbert state space of the system.

In terms of the above variables the quantization of the model is defined by the non vanishing canonical brackets

$$
\begin{aligned}
& {\left[x^{\mu}, b_{v}\right]=i \delta_{\mu \nu}} \\
& \left\{t^{a}, \bar{\psi}^{b}\right\}=\delta_{a b}
\end{aligned}
$$

The bosonic operators are self-adjoint with respect to a suitably defined scalar product. We will be working in the "coordinate" representation of the Schrödinger picture, with the states described by wave functions $\phi(x, \xi, t)$, where $\xi$ collectively denotes the diagonal fermionic coordinates. Correspondingly, our choice for the scalar product is

$$
\left\langle\phi_{1}, \phi_{2}\right\rangle=\Sigma_{\xi} \int d^{N} \times \phi_{1}^{*}(x, \xi, t) \phi_{2}(x, \xi, t)
$$

and the absence of the $\sqrt{g}$ factor in the integration should be noted. If use of the invariant measure $\sqrt{g} d^{N} x$ is preferred, one has to work with operators $\hat{O}$ related to ours by the similarity relation

$$
\hat{0}=g^{-1 / 4} 0 g^{1 / 4} \text {. }
$$

The self-adjoint momentum $p_{\mu}$ can be represented by $-i \partial / \partial x^{\mu}:$ this is possible because of the choice of the integration measure and because our fermionic states are built by application of the $\bar{\psi}^{a}$ operators, which commute with $p_{\mu}$. These points are further elucidated in Appendix $B$.

Next we recall that SUSY suggested the two sets of basic canonical variables $\Omega_{1}$ and $\Omega_{2}$. In order to identify the quantum forms of the variables $P_{\mu}^{(1)}$ and $P_{\mu}^{(2)}$, we introduce the quantized version of eqs. (3.17). In such a procedure we face an ordering ambiguity. since $\psi^{a}$ and $\bar{\psi}^{a}$ do not anticommute after quantization, these operators can be ordered in many ways in the r.h.s. of eqs. (3.17) and, as we shall see, each choice is consistent with supersymmetry. 
$-16-$

Now we choose to quantize those equations as follows:

$$
\begin{aligned}
& p_{\mu}^{(1)}-p_{\mu}=-\frac{i}{2} e_{a}^{\nu} \partial_{\mu} e_{\nu b}\left[\psi^{a}, \bar{\psi}^{b}\right]+i \partial_{\mu} w, \\
& p_{\mu}^{(2)}-p_{\mu}=\frac{i}{2} e_{b}^{v} \partial_{\mu} e_{\nu a}\left[\psi^{a}, \bar{\psi}^{b}\right]-i \partial_{\mu} w,
\end{aligned}
$$

and as a consequence

$$
\begin{gathered}
p_{\mu}^{(2)}-p_{\mu}^{(1)}=\frac{i}{2} \partial_{\mu} g_{\lambda_{s}}(x)\left[\psi^{\lambda}, \bar{\psi}^{s}\right]-2 i \partial_{\mu} w . \\
\text { These definitions imply } \\
p_{\mu}^{(1)+}=p_{\mu}^{(2)}
\end{gathered}
$$

(while $p_{\mu}^{+}=p_{\mu}$ )

An important remark is that if we choose a different ordering, for instance by substituting in eqs. (4.4)

$$
\left[4^{a}, \bar{\psi}^{b}\right] \rightarrow 24^{a} \bar{\psi}^{b},
$$

we can reobtain the form $(4.4)$ by means of the substitution

$$
W(x) \rightarrow \tilde{W}(x)=W(x)-1 / 2 \ln e(x)
$$

where $e(x) \equiv \operatorname{det}\left[e_{\mu}^{a}(x)\right]$.

It is thus clear that any ordering is consistent with SUSY, since the substitution (4.6) does not affect the supersymetry properties of the model.

The difference however will show up when we shall consider invariance under general coordinate transformations, as it will be done in the next chapter. Under G.C.T., ln e transforms differently from the (scalar) potential $w(x)$. As a consequence, we anticipate that only the combination of SUSY and G.C.T. removes this ambiguity and leads to the unique choice given by ens. (4.4).

From eqs. (4.4) it follows that each of the two sets $\Omega_{1}$ and $\Omega_{2}$ satisfies a set of canonical commutation relations whose non vanishing elements are 


$$
\begin{aligned}
& {\left[x^{\mu}, p_{\nu}(1)\right]=i \delta_{\mu \nu},} \\
& \left\{\psi^{\mu}, \bar{q}_{\nu}\right\}=\delta_{\mu \nu}
\end{aligned}
$$

and

$$
\begin{aligned}
& {\left[x^{\mu}, p_{\nu}{ }^{(2)}\right]=i \delta_{\mu \nu},} \\
& \left\{\bar{\psi}^{\mu}, \psi_{\nu}\right\}=\delta_{\mu \nu} .
\end{aligned}
$$

This fact corresponds to the existence of the canonical Poisson brackets for the sets $\Omega_{1}$ and $\Omega_{2}$. The quantum transformations among the different sets of variables are the quantum counterpart of complex canonical transformations for the classical case; they are similarity transformations that preserve commutation relations but not the scalar products in the Hilbert space.

For the representation of $p_{\mu}^{(1)}$ and $p_{\mu}^{(2)}$ by differential operators in the Schroedinger picture consult App.B, where it is shown that, in the basis in which fermionic states are built by application of covariant $\bar{\psi}_{\mu}$ or controvariant $\bar{\psi}^{\mu}$ respectively, either $p_{\mu}^{(1)}$ or $p_{\mu}^{(2)}$ can be represented by the (non hermitean) operator $-i \partial \mu$.

The interest in the sets of variables $\Omega_{1}$ and $\Omega_{2}$ at the quantum level becomes evident when we consider the expressions for the SUSY charges $Q_{1}$ and $Q_{2}$, given by the classical formulae (3.8) and (3.9). Thanks to the relations

$$
\left[p_{\mu}^{(1)}, \psi^{V}\right]=0, \quad\left[p_{\mu}^{(2)}, \bar{\psi}^{\nu}\right]=0
$$

no ambiguity arises in quantizing (3.8) and (3.9) and the quantum charges are simply given by

$$
\begin{aligned}
& Q_{1}=p_{\mu}^{(1)} \psi^{\mu} \\
& Q_{2}=p_{\mu}^{(2)} \bar{\psi}^{\mu}
\end{aligned}
$$

The formulae (4.9) are thus devoid of ordering ambiguities. 
$-18-$

As a consequence of the commutation relations we find

$$
Q_{1}^{2}=Q_{2}^{2}=0
$$

Finally the quantum Hamiltonian will be defined as the suSY invariant expression

$$
H=\frac{1}{2}\left\{Q_{1}, Q_{2}\right\}
$$

In order to proceed further and to compare with other works on the subject, it is convenient (as in sect.3) to introduce the quantum operator given by:

$$
\begin{aligned}
\pi_{\mu} & =p_{\mu}^{(1)}-\frac{i}{2} T_{\mu \nu}^{\lambda}\left[\bar{\psi} \lambda, \psi^{v}\right]-i \partial_{\mu} w, \\
& =p_{\mu}^{(2)}-\frac{i}{2} T_{\mu \nu}^{\lambda}\left[\psi \lambda, \bar{\psi}^{v}\right]+i \partial_{\mu} w, \\
& =p_{\mu}+\frac{i}{2} \omega_{\mu, a b}\left[\bar{\psi}^{a}, \psi^{b}\right] .
\end{aligned}
$$

starting from eqs.(4.9) it is then easy to prove that

$$
\begin{aligned}
& Q_{1}=\frac{1}{2}\left\{\psi^{\mu}, \pi_{\mu}\right\}+\frac{i}{2}\left\{\psi^{\mu}, \partial_{\mu} w\right\}, \\
& Q_{2}=\frac{1}{2}\left\{\bar{\psi}^{\mu}, \pi_{\mu}\right\}-\frac{i}{2}\left\{\bar{\psi}^{\mu}, \partial_{\mu} w\right\} .
\end{aligned}
$$

A convenient way of expressing eqs.(4.13) is obtained by introducing

$$
\begin{aligned}
& \pi_{\mu}^{+}=g^{1 / 4} \pi_{\mu} g^{-1 / 4}=\pi_{\mu}+i \partial_{\mu} \ln g^{1 / 4}, \\
& \pi_{\mu}^{-}=g^{-1 / 4} \pi_{\mu} g^{1 / 4}=\pi_{\mu}-i \partial_{\mu} \ln g^{1 / 4},
\end{aligned}
$$

and corresponding ty

$$
\begin{aligned}
& Q_{1}=\psi^{\mu}\left(\pi_{\mu}^{+}+i \partial_{\mu} w\right)=\left(\pi_{\mu}^{-}+i \partial_{\mu} w\right) \psi^{\mu}, \\
& Q_{2}=\psi^{\mu}\left(\pi_{\mu}^{+}-i \partial_{\mu} w\right)=\left(\pi_{\mu}^{-}-i \partial_{\mu} w\right) \bar{\psi}^{\mu} .
\end{aligned}
$$


Performing the fundamental anticommutator (4.11) and using eqs. (4.15) we finally obtain for $H$ the expression

$$
H=t_{0}+V
$$

where

$$
\begin{aligned}
& H_{0}=\frac{1}{2} \pi_{\mu}^{-} g^{\mu \nu}(x) \pi_{\nu}^{+} \\
& V=\frac{1}{2} g^{\mu \nu}(x) D_{\mu} W D_{v} w+\frac{1}{2} D_{\mu} D_{v} W\left[\Psi^{\mu}, \psi^{\nu}\right]+ \\
& +\frac{1}{8} R_{\mu v \lambda_{s}}\left[\Psi^{\mu}, \psi^{v}\right]\left[\bar{\psi}^{\lambda}, \psi^{s}\right] .
\end{aligned}
$$

Computing

$$
\hat{H}=g^{-1 / 4}+H g^{1 / 4}
$$

we find complete agreement in form and in ordering with the Hamiltonian given in Ref.[12]. The main point to be discussed remains the ordering property of eqs. (4.17). This will be done in sect. 6 . 
5. GENERAL COORDINATE TRANSFORMATIONS .

In the previous discussion we have stressed the relevance of G.C.T. in order to solve all ordering ambiguities and we have anticipated that the choice $(4.4)$ is actually imposed by G.C.T. requirements. In this section we shall examine the quantum formulation of G.C.T. and finally show that the Hamiltonian $\mathrm{H}\left(x^{\mu}, \mathrm{p}_{\mu} ; \psi^{a}, \bar{\Psi}^{a}\right)$ is indeed invariant. Our proof will make essential use of the two sets of basic variables $\Omega_{1}$ and $\Omega_{2}$ introduced earlier.

An infinitesimal G.C.T. is written as

$$
x^{\mu} \rightarrow x^{\mu}=x^{\mu}+f^{\mu}(x)
$$

or

$$
\Delta x^{\mu}=x^{\mu}-x^{\mu}=f^{\mu}(x)
$$

where $f_{\mu}(x)$ is infinitesimal.

Under (5.1) $\psi^{a}$ and $\bar{\psi}^{a}$ are scalar quantities

$$
\Delta \psi^{a}=\Delta \Psi^{a}=0
$$

So, the generator $G$ of unitarily implemented G.C.T. does not contain fermionic variables and therefore has the same form as in the non supersymmetric case and is uniquely given by [1]

$$
G=\frac{1}{2}\left\{p_{\mu}, f^{\mu}\right\}
$$

As a consequence we shall write for the total variation $\triangle A$ of a dynamical variable $A$

$$
\Delta A=i[G, A]+\delta A
$$

where $\delta_{A}$ denotes the variation in the functional form of $A$ due to the dependence on $e_{\mu}^{a}, e^{\mu} \alpha, W J$ 
$-21-$

The variation $\Delta \xi$ of the fundamental variables $\xi \equiv\left(x^{\mu}, b_{\mu}, 4^{a}, \overline{4}^{a}\right)$ of the set $\Omega$ are indeed given by

$$
\Delta \xi \geq i[\sigma, \xi] \text {. }
$$

In order to proceed further and to evaluate the complete variation of a dynamical variable $\mathrm{A}\left(x^{\mu}, p_{\mu}, \psi^{a}, \bar{\Psi}^{a}\right)$ we have to take into account that in general $A$ is a functional of the quantities $e_{\mu}, e_{a}^{\mu}$ and $w$ also. The form of these quantities is changed by a G.C.T. according to the laws

$$
\begin{aligned}
& \delta e_{\mu}^{a}=e_{\mu}^{a}(x)-e_{\mu}^{a}(x)=e_{\lambda}^{a}(x) \partial_{\mu} f^{\lambda}(x)-\partial_{\lambda} e_{\mu}^{a}(x) \cdot f^{\lambda}(x),(5.6 \mathrm{a}) \\
& \delta e_{a}^{\mu}=e_{a}^{\lambda}(x) \partial_{\lambda} f^{\mu}(x)-\partial_{\lambda} e_{a}^{\mu}(x) \cdot f^{\lambda}(x), \\
& \delta\left(e_{a}^{\nu} \partial_{\mu} e_{v b}\right)=-e_{a}^{\nu} \partial_{\lambda} e_{\nu b} \cdot \partial_{\mu} f^{\lambda}(x)-(5.6 \mathrm{~b}) \\
& -e_{a}^{\nu} e_{\lambda b} \partial_{\mu} \partial_{\nu} f^{\lambda}(x)-\partial_{\lambda}\left(e_{a}^{\nu} \partial_{\mu} e_{\nu b}\right) \cdot f^{\lambda}(x), \\
& \delta e=\delta\left(\operatorname{det} e_{\mu}^{a}(x)\right)=-e \partial_{\mu} f^{\mu}(x)-\partial_{\lambda} e \cdot f^{\lambda}(x), \text { (5.6d) } \\
& \delta W=-\partial_{\lambda} w \cdot f^{\lambda}(x) .
\end{aligned}
$$

We are now in the position to show that

$$
\Delta H\left(x^{\mu}, b_{\mu}, 4^{a}, \Psi^{a}\right)=0
$$

which will be achieved by proving that

$$
\Delta Q_{1}=0, \Delta Q_{2}=0 \text {. }
$$

In order to obtain this result we shall make constant use of the variables of the sets $\Omega_{1}$ and $\Omega_{2}$, which, as already stressed, allow a simple and unambiguous expression of the generators $Q_{1}$ and $Q_{2}$.

The proof will be made easier by the introduction of the two auxiliary operators

$$
\begin{aligned}
G_{1}= & \frac{1}{2}\left\{Q_{1}, f^{\mu} \bar{\psi}_{\mu}\right\}=\frac{1}{2}\left\{f f^{\mu}(x), p_{\mu}^{(1)}\right\}- \\
& -\frac{1}{2} \partial_{\nu} f^{\mu}(x)\left\{\psi^{v}, \Phi_{\mu}\right],
\end{aligned}
$$


$-22-$

$$
\begin{aligned}
G_{2}= & \frac{1}{2}\left\{Q_{2}, f^{\mu} \psi_{\mu}\right\}=\frac{1}{2}\left\{f^{\mu}(x), p_{\mu}^{(2)}\right\}- \\
& -\frac{i}{2} \partial_{\nu} f^{\mu}(x)\left[\bar{\psi}^{\nu}, \psi_{\mu}\right],
\end{aligned}
$$

where we have used the basic definitions of $Q_{1}$ and $Q_{2}$

$$
Q_{1}=p_{\mu}^{(1)} \psi^{\mu}, Q_{2}=p_{\mu}^{(2)} \Psi^{\mu} \text {. }
$$

By using the fact that $Q_{1}$ and $Q_{2}$ are nilpotent, we have that

$$
\begin{aligned}
& {\left[Q_{1}, G_{1}\right]=0,} \\
& {\left[Q_{2}, G_{2}\right]=0 .}
\end{aligned}
$$

The next step is to use the quantum definitions of $p_{\mu}^{(1)}, p_{\mu}^{(2)}$ given in eqs.(4.3). This leads us finally to the fundamental separation

$$
G_{1,2}=G+X_{1,2}
$$

where $x_{1}$ and $x_{2}$ are

$$
\begin{aligned}
X_{1}= & -\frac{i}{2}\left[\psi^{a}, \bar{\psi}^{b}\right]\left(f^{\mu} e_{a}^{\nu} \partial_{\mu} e_{\nu b}+\partial_{\mu} f^{\nu} \cdot e_{a}^{\mu} e_{v b}\right)+ \\
& +i f^{\mu} \partial_{\mu} W, \\
X_{2}= & \frac{i}{2}\left[\psi^{a}, \bar{\psi}^{b}\right]\left(f^{\mu} e_{b}^{\nu} \partial_{\mu} e_{\nu a}+\partial_{\mu} f^{\nu} e_{b}^{\mu} e_{\nu a}\right) \\
& -i f^{\mu} \partial_{\mu} W .
\end{aligned}
$$

The importance of $x_{1}$ (and $x_{2}$ ) lies in the fact that the commutators of $x_{1}\left(x_{2}\right)$ with the variables of the set $\Omega_{1}\left(\Omega \Omega_{2}\right)$ coincide with the variation in form of those variables considered as functions of the variables of the set $\Omega$, namely

$$
\delta \xi_{i}=i\left[X_{i}, \xi_{i}\right], \quad i=1,2 \text {. }
$$

Indeed a direct computation (based on the use of eqs.(5.6)) shows that 
$-23-$

$$
\begin{aligned}
i\left[x_{1}, \psi^{\mu}\right] & =\psi^{a}\left(e_{a}^{\nu} \partial_{\nu} f^{\mu}-f^{\nu} \partial_{\nu} e^{\mu} a\right)= \\
& =\psi^{a} \delta e_{a}^{\mu}=\delta \psi^{\mu},
\end{aligned}
$$

$$
\begin{aligned}
i\left[x_{1}, \Psi_{\mu}\right] & =-\Psi^{a}\left(e_{a}^{\nu} \partial_{\mu} f_{\nu}+f^{\nu} \partial_{\nu} e_{\mu a}\right)= \\
& =\Psi^{a} \delta e_{\mu a} \equiv \delta \Psi_{\mu}
\end{aligned}
$$

$$
\begin{aligned}
& i\left[x_{1}, p_{\mu}^{(1)}\right]=-i \partial_{\mu}\left(f^{\lambda} \partial_{\lambda} w\right)+\frac{i}{2}\left[\psi^{\nu}, \Psi_{\lambda}\right] \partial_{\mu} \partial_{\nu} f^{\lambda}+(5.13 c) \\
& +\frac{i}{2}\left[\psi^{\alpha}, \Psi_{b}\right]\left(e_{\alpha}^{\nu} \partial_{\lambda} e_{\nu b} \partial_{\mu} f^{\lambda}+f^{\lambda} \partial_{\lambda} e_{\alpha}^{\nu} \partial_{\mu} e_{\nu b}\right)=\delta b_{\mu}^{(1)}
\end{aligned}
$$

As a consequence we find that the total variation of any variable $\xi_{1}$ of $\Omega_{1}$ can be written as

$$
\begin{aligned}
\Delta \xi_{1} & =i\left[G_{1} \xi_{1}\right]+\delta \xi_{1}=i\left[G+X_{1}, \xi_{1}\right]= \\
& \equiv i\left[G_{1}, \xi_{1}\right]
\end{aligned}
$$

By the same chain of arguments one can easily obtain that any variable $\xi_{2}$ of the set $\Omega_{2}$ verifies

$$
\Delta \xi_{2}=i\left[G_{2}, \xi_{2}\right] \text {. }
$$

A glance at all these results shows that there is a correspondence between each operator $G, G_{1}, G_{2}$ and the three sets of variables $\Omega, \Omega_{1}, \Omega_{2}$. Indeed, each of the $G, G_{1}, G_{2}$ generates a total transformation $\Delta$ when commuted with the quantities $\xi_{1} \xi_{1}, \xi_{2}$ respectively.

Eqs. (5.14) and (5.15) represent the key result in our proof of the G.C.T. invariance of the Hamiltonian. By applying it to the supersymmetric generator $Q_{1}$ we have

$$
\begin{aligned}
& \Delta Q_{1}=i\left[G_{1}, Q_{1}\right], \\
& \Delta Q_{2}=i\left[G_{2}, Q_{2}\right] .
\end{aligned}
$$

Using now eds. (5.9) we reach the conclusion that

$$
\Delta Q_{1}=\Delta Q_{2}=0 \text {. }
$$


The supersymmetric representation for $H$, eq. (4.11), leads finally to the announced result $\Delta H=0$, the invariance of the Hamiltonian under G.C.T.

It is important to remark that all our arguments are based on the transformation properties of $e_{\mu}^{a}$ and $\omega$ given in eqs. (5.6). Had we chosen a different ordering in the definitions of $b_{\mu}^{(1)}$ and $p_{\mu}^{(2)}$ all the equations would have remained the same apart from, the replacement of $W(x)$ by $W(x)$ :

$$
\tilde{w}(x)=w(x)-\frac{1}{2} \ln e(x) \text {. }
$$

Now (see eqs.5.6)

$$
\delta \widetilde{w}(x)=-f^{\mu} \partial_{\mu} \tilde{w}(x)-\frac{1}{2} \partial_{\mu} f^{\mu} .
$$

The extra term would show up in our calculation and yield $\Delta Q_{1} \neq 0, \Delta Q_{2} \neq 0$. We thus realize that the choice of ordering made in eqs. (4.4) is the only one which guarantees general reparametrization invariance.

An alternative way of proving invariance under G.C.T. is suggested in the second part of App.B, where it is shown that

$$
\Delta \pi_{\mu}^{+}=-\partial_{\mu} f^{\lambda}(x) \cdot \pi_{\lambda}^{+}, \Delta \pi_{\mu}^{-}=-\pi_{\lambda}^{-} \partial_{\mu} f^{\lambda}(x) .
$$

As a consequence both $H_{0}$ and $V$ as given by eqs.(4.17) are separately invariant under G.C.T. This is completely analogous to what happens in the purely bosonic case, eq.(1.6). As in that case, pure invariance under G.C.T. doesn't fix uniquely the appropriate Hamiltonian. A unique determination is only given by combination of G.C.T. and SUSY invariance requirements. 
$-25-$

6. THE ORDERING PROPERTY

Let us refer explicitly to the form of the Hamiltonian given in eq. (4.17): it is important to notice that the discussion leaves open the choice of the operator basis $\left(\Omega, \Omega_{1,2}\right)$ to be used to express $H$. Indeed we shall see that the ordering properties will not be the same in the three basic sets.

We first consider the set $\Omega\left(x^{\mu}, b_{\mu} ; \psi^{a}, \bar{\psi}^{a}\right)$ (i) One can identify two sources of deviation from Weyl ordering (W.O.) ${ }^{(*)}$.

I) The purely bosonic term can be written as

$$
\begin{gathered}
\frac{1}{2} g^{-1 / 4} p_{\mu} g^{1 / 2} g^{\mu \nu} p_{\nu} g^{-1 / 4}=\frac{1}{2}\left(p_{\mu} p_{\nu} g^{\mu \nu}\right) w_{0}+ \\
+\frac{1}{8}\left(R+g^{\nu \sigma} \Gamma_{\nu \lambda}^{\mu} \Gamma_{\mu \sigma}^{\lambda}\right),
\end{gathered}
$$

thus leading to a deviation

$$
\Delta V_{I}=\frac{1}{8}\left(R+g^{\nu \sigma} \Gamma_{\nu \lambda}^{\mu} \Gamma_{\mu \sigma}^{\lambda}\right)
$$

II) The four fermion term reads

$$
\begin{aligned}
& -\frac{1}{16}\left(g^{\mu \nu} \omega_{\mu, a b} \omega_{\nu, c d}-R_{a b c d}\right) \cdot \\
& \cdot\left\{\left[\Psi^{a}, \psi^{b}\right],\left[\bar{\psi}^{c}, \psi^{d}\right]\right\} .
\end{aligned}
$$

Using the ordering identity

$$
\frac{1}{8}\left\{\left[\bar{\psi}_{A}, \psi_{B}\right],\left[\bar{\psi}_{C}, \psi_{D}\right]\right\}=\left(\bar{\psi}_{A} \bar{\psi}_{C} \psi_{B} \psi_{D}\right)_{W_{D}}-\frac{1}{4} \delta_{A D} \delta_{B C}{ }^{(6.4)}
$$

we obtain a deviation

$$
\Delta v_{I I}=\frac{1}{8}\left(-R+g^{\mu \nu} \omega_{\mu, a b} \omega_{\nu, a b}\right) \text {. }
$$

Hence the overall deviation is

(*) General properties of $W .0$. and some details of the computations are provided in appendix $A$. 


$$
\Delta V=\Delta V_{I}+\Delta V_{I I}=\frac{1}{8} g^{\mu \nu}\left(\Gamma_{\mu \lambda}^{\sigma} \Gamma_{V \sigma}^{\lambda}+\omega_{\mu, a b} \omega_{\mu, a b}\right) .
$$

We thus see that although the terms in $R$ cancel, a non vanishing contribution is left: the conclusion is that the Hamiltonian expressed in the variables $\Omega$ is not weyl ordered.

Let us now make use of the variables $\Omega_{\mu}\left(x^{\mu}, p_{\mu}^{(1)}, 4^{\mu}, i \bar{\psi}_{\mu}\right)$. Again the following deviations from Weyl ordering are found.

I) The purely bosonic term

$$
\frac{1}{2} g^{-1 / 4} p_{\mu}^{(1)} g^{\mu \nu} p_{v}^{(1)} g^{-1 / 4}
$$

can be treated in the same way as before and leads to the deviation

$$
\Delta V_{I}^{(1)}=\frac{1}{8}\left(R+q^{v \sigma} \Gamma_{\nu \lambda}^{\mu} \Gamma_{\mu \sigma}^{\lambda}\right)
$$

II) For the four-fermion piece

$$
\frac{1}{16}\left(R_{\lambda \rho \sigma \sigma}-g^{\mu \nu} \Gamma_{\lambda, \mu \rho} \Gamma_{\sigma, v \tau}\right)\left\{\left[\bar{\psi}^{\lambda}, \psi^{\rho}\right],\left[\bar{\psi}^{\sigma}, \psi^{\varepsilon}\right]\right\}
$$

we find, via eq. $(6.4)$, the deviation

$$
\Delta V_{\text {II }}^{(1)}=-\frac{1}{8}\left(R+g^{\mu v} \Gamma_{\mu \rho}^{\lambda} \Gamma_{\nu \lambda}^{\rho}\right)
$$

Combining the results $(6.7)(6.8)$ we achieve a complete cancellation i.e.

$$
\Delta V_{I}^{(1)}+\Delta V_{\underline{I}}^{(1)} \equiv \Delta V^{(1)}=0
$$

Hence the Hamiltonian written in terms of the variables $\Omega_{1}$ (or $\Omega_{2}$ ) is weyl ordered.

This result may look somewhat surprising, even if the fact that things are different for different sets of variables is not unexpected, owing to the presence of a non linear transformation between $\Omega$ and $\Omega_{1}$. In order to clarify why $\Omega_{1}$ and $\Omega_{2}$ are privileged, we offer an alternative derivation of the same result, based on the simple behaviour of the $\Omega_{1}$ and $\Omega_{2}$ variables under sUSY transformations. 
$-27-$

Our strategy will be to trace the ordering properties of $\mathrm{H}$ from those of the simpler operators $Q_{1}$ and $Q_{2}$. Let us thus express $Q_{2}=p_{\mu}$ (2) $4 \mu$ in terms of the variables $\Omega_{1}$. It is easy to check that $Q_{2}$ is Weyl ordered:

$$
\begin{aligned}
Q_{2} & =\left(g^{\mu \nu} \Psi_{\nu} p_{\mu}^{(1)}\right)_{w_{0}}-i\left(\Psi_{\lambda} g^{\mu \lambda} \partial_{\mu} w\right)_{w_{0}}+ \\
& +i\left(\bar{\psi}_{\sigma} \bar{\Psi}_{\lambda} g^{\mu \lambda} g^{\rho \sigma} \partial_{\mu} g_{\gamma_{g}} \psi^{\nu}\right)_{w_{0}}
\end{aligned}
$$

Next we compute $H$ through the fundamental anticomutator of $Q_{1}$ and $Q_{2}$. In such calculation we are indeed helped by the great simplicity of $Q_{1}$ in the variables $\Omega_{1}$.

This simplicity becomes apparent if one adopts a useful device which simplifies the calculation and explains immediately why $\mathbf{w} .0$. is preserved. The Hamiltonian $\mathbf{H}$ can be most conveniently rewritten by introducing an anticommuting parameter $\varepsilon$ as follows:

$$
Q_{2}(\varepsilon)=e^{1 / 2 \varepsilon Q_{1}} Q_{2} e^{-1 / 2 \varepsilon Q_{1}}=Q_{2}+\frac{\varepsilon}{2}\left\{Q_{1}, Q_{2}\right\}
$$

Then

$$
H=\int d \varepsilon Q_{2}(\varepsilon)
$$

By inserting into eq. (6.10) the expression for $Q_{2}$ provided in $(6.9)$, one easily ascertains that $Q_{2}(\varepsilon)$ can be expressed in terms of the linear combinations

$$
\begin{aligned}
& x^{\mu}(\varepsilon)=e^{1 / 2 \varepsilon Q_{1}} x^{\mu} e^{-1 / 2 \varepsilon Q_{1}}=x^{\mu}+\varepsilon \psi^{\mu}, \\
& \Psi_{\mu}(\varepsilon)=e^{1 / 2 \varepsilon Q_{1}} \Psi_{\mu} e^{-1 / 2 \varepsilon Q_{1}}=\Psi_{\mu}+\varepsilon p_{\mu}{ }^{(1)}, \\
& P_{\mu}(\varepsilon)=P_{\mu}{ }^{(1)}, \\
& \psi^{\mu}(\varepsilon)=\psi^{\mu} .
\end{aligned}
$$

It is important to notice that each of the expression (6.12) linearly combines only commuting operators; these combinations therefore obey the same 
$-23-$

commutations relations as the original variables. This entails that $Q_{2}(\varepsilon)$ is w.o. when expressed through the variables (6.12), since the original $Q_{2}$ is w.o. in the basic variables $\Omega_{1}$.

Finally from eqs. $(6.10)(6.11)$ we obtain

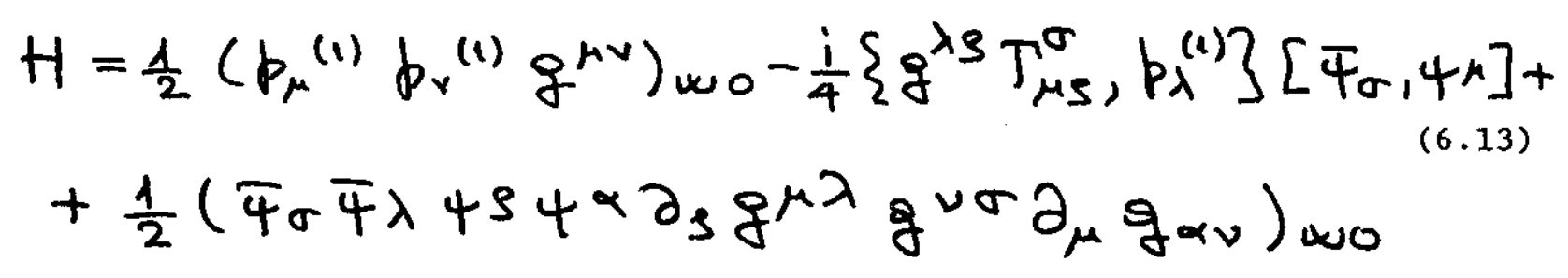

It is instructive to verify again the absence of $W .0$. by inserting the variables $\Omega$ in eq. (6.13). One sees that the only deviation from wo. comes from the new four-fermion term introduced by the nonlinear transformation between $P_{\mu}^{(1)}$ and $P_{\mu}$ This is

$$
\begin{aligned}
& -\frac{1}{16}\left(e_{\alpha}^{\alpha} \partial_{\mu} e_{\alpha b} e_{c}^{\beta} \partial_{\nu} e_{\beta d} g^{\mu \nu}+\right. \\
& \left.+g^{\lambda \gamma} e_{\sigma c} e^{\beta} \Gamma_{\beta \gamma}^{\sigma} e_{a}^{\alpha} \partial_{\lambda} e_{\alpha b}\right)\left\{\left[\psi^{a}, \bar{\psi}^{b}\right],\left\{\psi^{c}, \bar{\psi}^{d}\right]\right\}
\end{aligned}
$$

and leads via eq. $(6.4)$ to the deviation

$$
\begin{aligned}
\Delta V & =-\frac{1}{8}\left(e^{\alpha} \partial_{\mu} e_{\alpha b} e^{\beta} \partial_{v} e_{p a} g^{\mu \nu}+\right. \\
& \left.+g^{\lambda \gamma} e \sigma b e^{\beta} \Gamma_{\beta \gamma}^{\sigma} e^{\alpha} \partial_{\lambda} e_{\alpha b}\right) .
\end{aligned}
$$

Using eq.(2.21) one can verify the identity between the expressions (6.14) and (6.6) for $\Delta V$ in the variables $\Omega$. 


\section{CONCLUSIONS}

We have seen that the combined use of SUSY and G.C.T. invariance fixes the ordering in the Hamiltonian in a totally unique way. Our treatment makes clear that both requirements are needed: SUSY alone leaves still some ambiguity.

we stress that our two sets of variables $\Omega_{1}$ and $\Omega_{2}$ play a very privileged role throughout the whole discussion. Actually, these variables are canonical and appear in the two SUSY charges in a remarkably simple way. As a consequence, they are instrumental in proving the G.C.T. invariance of the whole approach. On the other hand, we have shown that our reparametrization invariant Hamiltonian is Wey1-ordered when expressed in terms of those variables. It is rather striking that the role of the sets $\Omega_{1}$ and $\Omega_{2}$ is unique in this respect: when transforming to the familiar variables $\Omega$ -which are suggested by hermiticity (and are not simple with respect to SUSY)Weyl ordering in the Hamiltonian is lost.

It is amusing to speculate on the implications of our result in the context of the path-integral approach. The equivalence between weyl-ordering and middle-point prescription is well-known in the bosonic case [3]. Although we have not been able to find an analogous statement in the literature for the fermionic case, we are convinced that the equivalence can also be extended to this case. As a consequence, it seems reasonable to assess that in the SUSY case the path-integral method can be straightforwardly applied to the G.C.T. invariant Hamiltonian with the middle-point prescription for bosons and fermions, provided either the set $\Omega_{1}$ or $\Omega_{2}$ is used as integration variables. The reason is due to the striking boson-fermion cancellation given by eqs. (6.7) (6.8), which implies that no further effective potential has to be added to the Hamiltonian. This situation should be contrasted with what happens by expressing our reparametrization-invariant Hamiltonian in terms of the variables $\Omega$, where - owing to eq.(6.6) - the modified Hamiltonian

$$
H+\Delta V=H^{\prime}
$$

should be used in the path-integral. As Gervais and Jewicki have shown [5], an analogous situation occurs in the non SUSY case.

A further use of the variables $P_{\mu}^{(1)}, P_{\mu}^{(2)}$ is related to the simple form of the supersymmetric wave functions for the ground states of the model corresponding to the empty $\left(\psi_{0}\right)$ and filled $\left(\psi_{F}\right)$ sectors of the fermion Fock space. Indeed the beautiful result given in Ref. [12] 


$$
\psi_{0} \sim e^{-w} g^{1 / 4}, \psi_{F} \sim e^{w} g^{-1 / 4}
$$

can be obtained with amusing simplicity by requiring that those states are annihilated by $p_{\mu}^{(1)}$ and $p_{\mu}^{(2)}$ respectively

$$
p_{\mu}^{(2)}|0\rangle=0, \quad p_{\mu}^{(1)}|F\rangle=0
$$

In this sense $p_{\mu}^{(2)}$ and $p_{\mu}^{(1)}$ can be considered as the supersymmetric generalization of the celebrated annihilation and creation operators for the harmonic oscillator [13].

We believe that the method proposed here is particularly suitable to extend the present discussion to supersymetric theories in more than one dimension. The two-dimensional case has become particularly relevant, due to the renewed interest in string models. It has been shown by Maharana and Veneziano [8] that the existence of Ward identities in the Fradkin-Polyakov approach to the string depends on the possibility of performing general canonical transformations in the path-integral. We hope to come back to this interesting question in the near future. 
$-31-$

APPENDIX A

We wish to list some properties of Weyl ordering which are useful for our investigation; for the general definitions the reader can refer to the works quoted in Ref. [4].

We shall consider pairs of bosonic conjugate operators $q_{i}$ and $p_{j}$, and of fermionic conjugate operators $\psi_{\alpha}$ and $\bar{\psi}_{\beta}$. For sake of definiteness $\psi_{\alpha}$ will be identified as the coordinate and ${\overline{\Psi_{\beta}}}_{\beta}$ as the momentum.

When dealing with expressions containing a single bosonic or fermionic momentum, weyl ordered products coincide with anticommutators or commutators:

$\left(p_{i} f(q)\right)_{w_{0}}=\frac{1}{2}\left(p_{i} f(q)+f(q) p_{i}\right)_{1}$

$\left(\psi_{\alpha} \bar{\psi}_{\beta}\right)_{w_{0}}=\frac{1}{2}\left(\psi_{\alpha} \bar{\psi}_{\beta}-\bar{\psi}_{\beta} \psi_{\alpha}\right)_{1}$

$\left(\psi_{\alpha} \psi_{\beta} \bar{\psi}_{\gamma}\right)_{w_{0}}=\frac{1}{2}\left(\psi_{\alpha} \psi_{\beta} \bar{\psi}_{\gamma}+\bar{\psi}_{\gamma} \psi_{\alpha} \psi_{\beta}\right)$.

If there is one bosonic and one fermionic momentum, we simply have

$\left(p_{i} \bar{\psi}_{\alpha} f(q) \psi_{\beta}\right)_{\infty 0}=\frac{1}{4}\left(p_{i} f(q)+f(q) p_{i}\right)\left(\bar{\psi}_{\alpha} \psi_{\beta}-\psi_{\beta} \bar{\psi}_{\alpha}\right)$

We turn now to expressions containing either two bosonic or two fermionic momenta:

$\left(p_{i} b_{j} f(q)\right)_{w 0}=\frac{1}{4}\left(p_{i} b_{j} f+p_{i} f b_{j}+p_{j} f b_{i}+f p_{i} b_{j}\right)$

$\left(\bar{\Psi}_{\alpha} \bar{\psi}_{\gamma} \psi_{\beta} \psi_{\delta}\right)_{\omega 0}=\frac{1}{4}\left(\bar{\Psi}_{\alpha} \bar{\Psi}_{\gamma} \psi_{\beta} \psi_{\delta}+\bar{\Psi}_{\alpha} \psi_{\beta} \psi_{\delta} \Psi_{\gamma}+\right.$

(A.6)

$\left.+\bar{\psi}_{\gamma} \psi_{\delta} \psi_{\beta} \bar{\psi}_{\alpha}+\psi_{\beta} \psi_{\delta} \bar{\Psi}_{\alpha} \bar{\Psi}_{\gamma}\right)=\frac{1}{4}\left(\psi_{\beta} \psi_{\delta} \bar{\psi}_{\alpha} \bar{\Psi}_{\gamma}+\right.$

$\left.+\psi_{\beta} \bar{\Psi}_{\alpha} \bar{\Psi}_{\gamma} \psi_{\delta}+\psi_{\delta} \bar{\Psi}_{\gamma} \bar{\Psi}_{\alpha} \psi_{\beta}+\bar{\Psi}_{\alpha} \bar{\Psi}_{\gamma} \psi_{\beta} \psi_{\delta}\right)$

Two fundamental identities, valid in the bosonic and fermionic cases respectively, will also be needed. The first one is 
$-32-$

$$
\begin{aligned}
& g^{-1 / 4} p_{i} g^{1 / 2} g^{i j} p_{j} g^{1 / 4}=\left(p_{i} p_{j} g^{i j}\right)_{w o}+\Delta V, \\
& \Delta V=\frac{1}{4}\left[\partial_{i} \partial_{j} g^{i j}-4 g^{-1 / 4} \partial_{j}\left(g^{i j} g^{1 / 2} \partial_{i} g^{-1 / 4}\right)\right]
\end{aligned}
$$

and after a somewhat cumbersome calculation one obtains ${ }^{(*)}$

$$
\begin{aligned}
& \Delta V=\frac{1}{4}\left(R+g^{\lambda \beta} \Gamma_{v \lambda}^{\mu} \Gamma_{\mu s}^{\nu}\right) \\
& \text { For fermionic operators we shall rely on the identity } \\
& \frac{1}{8}\left\{\left[\bar{\Psi}_{\alpha}, \psi_{\beta}\right],\left[\psi_{\delta}, \bar{\Psi}_{\gamma}\right]\right\}=\left(\bar{\Psi}_{\alpha} \bar{\psi}_{\gamma} \psi_{\beta} \psi_{\delta} \delta\right)_{\text {wo }}- \\
& \quad-\frac{1}{4} \delta_{\alpha \delta} \delta_{\beta \gamma}
\end{aligned}
$$

which can be easily verified.

(*) The calculation is essentially the same as in Ref.[5]. The curvature term $R$ is absent there, since the case of flat space was investigated. 
$-33-$

APPENDIX B

I. Our aim is to discuss the realization of the momentum operators $p_{\mu}, p_{\mu}(1)$ $p_{\mu}^{(2)}, \pi_{\mu}$ on the states in the Hilbert space.

Let us start from the states

$$
|x, 0\rangle
$$

defined by

$$
\begin{aligned}
& \hat{x}^{\mu}|x, 0\rangle=x^{\mu}|x, 0\rangle, \\
& \psi^{\alpha}|x, 0\rangle=0 .
\end{aligned}
$$

( $\hat{X}$ denotes the coordinate operators).

The action of $p_{\mu}$ on these states is defined by

$$
p_{\mu}|x, 0\rangle=i \partial_{\mu}|x, 0\rangle
$$

We now introduce the "Vierbein" states with a definite number of vierbein fermion levels occupied:

$$
\left|x,\left\{a_{k}\right\}\right\rangle=\bar{\Psi} a_{1} \ldots \bar{\Psi} a_{k}|x, 0\rangle, \quad(0 \leq k \leq N)
$$

Since $P_{\mu}$ and $\bar{\psi}^{a}$ commute, we have as a consequence of (B.4) that

$$
p_{\mu}\left|x_{1}\left\{a_{k}\right\}\right\rangle=i \partial_{\mu}\left|x_{1}\left\{a_{k}\right\}\right\rangle \text {. }
$$

Thus on the wave functions

$$
\phi_{\left\{a_{k}\right\}}(x)=\left\langle\left\{a_{k}\right\}, x \mid \phi\right\rangle
$$

the operator $p_{\mu}$ is represented by $-i \partial_{\mu}$, a hermitian operator since we choose in the normalization integral the non invariant measure

$$
\langle\phi, x\rangle=\int d^{N} x \phi^{*}(x) x(x) .
$$


$-34-$

We wish now to determine another set of states on which the (non hermitian) operator $\bar{p}_{\mu}^{(1)}$ acts as $i \partial_{\mu}$, where $\bar{p}_{\mu}^{(1)}$ recalling eq. (4.4a) of the text, is given by

$$
\begin{aligned}
\bar{p}_{\mu}^{(1)} & =p_{\mu}^{(1)}+i \partial_{\mu} \ln g^{1 / 4}-i \partial_{\mu} w=g^{-1 / 4} p_{\mu}^{(1)} g^{1 / 4}-i \partial_{\mu} w \\
& =p_{\mu}+i e^{v} \partial_{\mu} e_{V a} \bar{t}^{b} \psi^{a} .
\end{aligned}
$$

Using (B.3) and (B.4) it follows then that

$$
F_{\mu}^{(1)}|x, 0\rangle=i \partial_{\mu}|x, 0\rangle
$$

Now let us define the "covariant" states

$$
\left|x,\left\{\mu_{k}\right\}\right\rangle=\overline{4}_{\mu_{1}} \ldots \bar{\psi}_{\mu_{k}}\left|x_{1} 0\right\rangle
$$

On these states, since $\left[\bar{p}_{\mu}(1), \bar{\psi}_{v}\right]=0$, the operator $\bar{p}_{\mu}^{(1)}$ is again represented by i $\partial_{\mu}$ :

$$
F_{\mu}^{(1)}\left|x,\left\{\mu_{k}\right\}\right\rangle=i \partial_{\mu} \mid x_{1}\left\{\mu_{k}\right\}>
$$

(Obviously, owing to our definition (B.8) of the norm, $\bar{p}_{\mu}^{(1)}$ is not hermitian).
A similar treatment can be given for

$$
\begin{aligned}
\bar{p}_{\mu}^{(2)} & =p_{\mu}^{(2)}-i \partial_{\mu} \ln g^{1 / 4}+i \partial_{\mu} w=g^{n / 4} p_{\mu}^{(2)} g^{-1 / 4}+i \partial_{\mu} w \\
& =p_{\mu}-i e^{v}{ }_{b} \partial_{\mu} e_{\nu a} \psi^{b} \psi^{a}
\end{aligned}
$$

If we introduce the "contravariant" states

$$
\left|x,\left\{\nu_{k}\right\}\right\rangle=\bar{\psi}^{r_{1}} \ldots \bar{\psi}^{\nu_{k}}|x, 0\rangle
$$

we obtain $\left(\left[\bar{F}_{\mu}^{(2)}, \bar{\psi}^{r}\right]=0\right)$

$$
\delta_{\mu}^{(2)}\left|x_{1}\{\nu \mu\}\right\rangle=i \partial_{\mu}\left|x_{1}\{\nu k\}\right\rangle \text {. }
$$

Now let us devote our attention to the representation of the operator (see eq.4.12 in the text) 
$-35-$

$$
\pi_{\mu}=g_{\mu \nu} \dot{x}^{\nu}=p_{\mu}+\frac{i}{2} \omega_{\mu, a b}\left[\bar{\psi}^{a}, \psi^{b}\right]
$$

As a consequence of the previous definitions we check that $\pi_{\mu}$ acts as a spinor covariant derivative on the vierbein-types states:

$$
\begin{aligned}
& \pi_{\mu}\left|x_{1}\left\{a_{k}\right\}\right\rangle=i \partial_{\mu}\left|x_{1}\left\{a_{k}\right\}\right\rangle+ \\
& +i \Sigma_{b} \omega_{\mu, a b}\left|x,\left\{b_{l}\right\}\right\rangle=i D_{\mu}^{a b}\left|x_{1}\left\{b_{l}\right\}\right\rangle
\end{aligned}
$$

Analogous sly, $\pi_{\mu}^{-}=g^{-1 / 4} \pi_{\mu} g^{1 / 4}$ acts as a covariant derivative on the covariant states:

$$
\begin{aligned}
& g^{-1 / 4} \pi_{\mu} g^{1 / 4}\left|x,\left\{\mu_{k}\right\}\right\rangle=i \partial_{\mu}\left|x_{1}\left\{\mu_{k}\right\}\right\rangle- \\
& -i \Sigma_{\mu^{\prime}} \Gamma_{\mu_{\mu k}}^{\mu^{\prime} 2}\left|x_{1}\left\{\mu_{l}^{\prime}\right\}\right\rangle=i D_{\mu}\left|x_{1}\left\{\mu_{k}\right\}\right\rangle .
\end{aligned}
$$

Finally $\pi_{\mu}^{+}=g^{1 / 4} \pi_{\mu} g^{-1 / 4}$ acts as the covariant derivative on the contravariant states.

II. At this point it is instructive to apply the formalism of sects to the study of the transformation properties of $p_{\mu}, p_{\mu}^{(1)}, p_{\mu}^{(2)}$ and $\pi_{\mu}$ under G.C.T. .

We have

$$
\Delta p_{\mu}=i\left[G, p_{\mu}\right]=-\frac{1}{2}\left\{p_{\nu}, \partial_{\mu} f^{\nu}\right\}
$$

One should notice however that $p_{\mu}$, like all the variables of the set $\Omega$, is not invariant under local transformations in the vierbeine indices $a, b$.

on the contrary the sets $\Omega_{1}, \Omega_{2}$ are invariant under that group, which means that we have not to worry about such transformations when dealing with $Q_{1}, Q_{2}$ (and $H$ ).

It turns out that the variables $p_{\mu}^{(4)}$ and $p_{\mu}^{(2)}$ obey more complicated rules of transformation ie.

$$
\Delta p_{\mu}^{(1)}=-p_{\lambda}^{(1)} \partial_{\mu} f^{\lambda}(x)+i \psi^{\nu} \bar{\psi}_{\lambda} \partial_{\mu} \partial_{\nu} f^{\lambda}(x)
$$


$-36-$

$\Delta p_{\mu}^{(2)}=-p_{\lambda}^{(2)} \partial_{\mu} f^{\lambda}(x)+i \bar{\psi}^{\lambda} \psi_{\nu} \partial_{\mu} \partial_{\lambda} f^{\nu}(x)$.

It is amusing to notice that $\pi_{\mu}$, corresponding to the covariant derivative on "vierbein" fermion states (eq.B.17), has the simple transformation law

$$
\Delta \pi_{\mu}=-\frac{1}{2}\left\{\pi_{\lambda}, \partial_{\mu} f^{\lambda}(x)\right\}
$$

while it is invariant under local $(a, b)$ rotations.

The vectors $\pi_{\mu}^{+}$and $\pi_{\mu}^{-}$also have the simple transformation law

$$
\begin{aligned}
& \Delta \pi_{\mu}^{+}=-\partial_{\mu} f^{\lambda}(x) \cdot \pi \lambda^{+} \\
& \Delta \pi_{\mu}^{-}=-\pi_{\lambda}^{-} \partial_{\mu} f^{\lambda}(x)
\end{aligned}
$$

From these formulae we see that $H_{0}$, as given in $(4.17 a)$, is separately invariant under reparametrizations. The same occurs for $V$. This means that any combination

$$
H_{0}+C V
$$

is invariant under G.C.T. The value $c=1$ is finally fixed by susY. 
- $37-$

APPENDIX C

A simple illustration for the fermion case of the connection between weyl ordering and the middle point rule in the path integral approach, can be offered by considering a single fermion mode. (For the formalism adopted in this Appendix we refer to the book by T.D. Lee [4]).

Let us consider the standard annihilation and creation operators $a, a^{+}$, with the anticommutation relations

$$
\begin{aligned}
& \{a, a+\}=1, \\
& \{a, a\}=\{a+, a+\}=0,
\end{aligned}
$$

and the occupation number

$N=a^{+} a$.

The states can be classified in terms of the eigenstates of $\mathrm{N}$ :

$$
N|0\rangle=0, N|1\rangle=|1\rangle \text {. }
$$

In order to define the coordinate representation for the fermion states, we introduce the anticommuting variable $z$ such that

$$
a=z, \quad a^{+}=d / d z \text {. }
$$

The wave functions are

$$
\begin{array}{ll}
\langle z \mid 0\rangle=z, & \langle z \mid 1\rangle=1, \\
\langle 0 \mid z\rangle=-1, & \langle 1 \mid z\rangle=z,
\end{array}
$$

and obey the completeness relations

$$
\begin{aligned}
& \int\langle\alpha \mid z\rangle\langle z \mid \beta\rangle d z=\delta_{\alpha \beta} \\
& \Sigma_{\alpha}\left\langle z_{1} \mid \alpha\right\rangle\left\langle\alpha \mid z_{2}\right\rangle=z_{2}-z_{1}=\delta\left(z_{1}-z_{2}\right) .
\end{aligned}
$$


$-33-$

We also introduce the "Fourier transform"

$$
f(k)=\int \exp (k z) F(z) d z
$$

in terms of the anticommuting momentum $k$. We have

$$
\int \exp \left[k\left(z_{1}-z_{2}\right)\right] d k=\delta\left(z_{1}-z_{2}\right)
$$

Let us now consider the Weyl ordered Hamiltonian

$$
H=\frac{1}{2}\left(a^{+} a-a a^{+}\right)
$$

for which it is easy to show that

$$
\left\langle z_{1}|H| z_{2}\right\rangle=\frac{z_{1}+z_{2}}{2}=\int \exp \left[k\left(z_{1}-z_{2}\right)\right](-k) \frac{z_{1}+z_{2}}{2} d k
$$

Then for the short time action one finds

$$
\left\langle z_{1}\left|e^{-i H \Delta t}\right| z_{2}\right\rangle=\int \exp \left[k\left(z_{1}-z_{2}\right)+i \Delta t k \frac{z_{1}+z_{2}}{2}\right] d k
$$

If we now notice that

$$
z=4, k=\bar{\psi}
$$

are the "classical" analogues of $a$ and $a^{+}$, eq.(c.10) can be rewritten in the form

$$
\int \exp \left[i \Delta t\left(-i \bar{\psi} \frac{\psi_{1}-\psi_{2}}{\Delta t}+H d\right)\right] d k
$$

where

$$
H_{d}=\bar{\psi} \frac{\psi_{1}+\psi_{2}}{2}
$$

We thus realize that $H_{d}$, corresponding to the weyl ordered expression (c.8), is indeed computed with the middle point prescription. This elementary example shows explicitly the connection between weyl ordering and the middle point recipe and hints at its general validity. 
REFERENCES

1. B.S. Dewitt, Phys.Rev. 85(1952)653;

M. Omote and H.Sato, Progress of Theor.Phys. 47(1972)1367;

for a general introduction see also the book by L.S.Schulman, "Techniques and Applications of Path Integration", J.Wiley (1981), ch.24.

2. B.S. Dewitt, Rev.Mod.Phys. 29(1957)377.

3. F.A.Berezin, Theor.Math.Phys. 6(1971)194.

4. H.Wey1, "Group Theory and Quantum Mechanics", Dover (1931);

for a review see for instance F.A.Berezin, Usp.Fiz.Nauk 132(1980)497;

T.D.Lee, "Particle Physics and Introduction to Field Theory", Harwood Publ. (1981).

5. J.L.Gervais and A.Jevicki, Nuc1.Phys. B110 (1976)93.

6. L.Brink, P. Di Vecchia and P.Howe, Nucl.Phys. B118 (1977)76.

A. Neveu and P.West, Phys.Lett.B 182(1986)343.

7. J.Maharana and G.Veneziano, Phys.Lett.B, 169(1986)177; Nuc1.Phys.B $283(1987) 126$.

8. A.M.Polyakov, Phys.Lett. 103B(1981) 207.

E.S.Fradkin and A.A.Tseyt1in, Nucl.Phys. B261(1985)1.

9. E.Witten, Nuc1.Phys. B188(1981)513.

10. A.Nakamura and F.Palumbo, Phys.Lett.B147(1984)96;

A.C.Davis, A.J.Macfarlane, P.C.Popat and J.W. van Holten, J.Phys.17A(1984)2945;

A.J.Macfarlane and P.C.Popat, J.Phys. 17A(1984)2955;

A.V.Smilga, "How to quantize supersymmetric theories", I.T.E.P. preprint $100(1985)$.

11. S.Weinberg, "Gravitation and Cosmology" H.Wiley (1972).

12. M.Claudson and M.B.Halpern, Nuc1.Phys. B250(1985)689.

13. V.De Alfaro, S.Fubini and G.Furlan; "Quantization, instantons and supersymmetry", ISAS preprint, Trieste 35/85/EP (1985), to be published in the E.S.Fradkin Festschrift, A.Hilger, Bristol. 